\title{
Cross-Layer Design for Video Transmission over Wireless Rician Slow-Fading Channels Using an Adaptive Multiresolution Modulation and Coding Scheme
}

\author{
Yong Pei ${ }^{1}$ and James W. Modestino ${ }^{2}$ \\ ${ }^{1}$ Computer Science and Engineering Department, Wright State University, Dayton, OH 45435, USA \\ ${ }^{2}$ Electrical and Computer Engineering Department, University of Miami, Coral Gables, FL 33124, USA
}

Received 22 August 2006; Accepted 13 April 2007

Recommended by Alex Kot

\begin{abstract}
We describe a multilayered video transport scheme for wireless channels capable of adapting to channel conditions in order to maximize end-to-end quality of service (QoS). This scheme combines a scalable H.263+ video source coder with unequal error protection (UEP) across layers. The UEP is achieved by employing different channel codes together with a multiresolution modulation approach to transport the different priority layers. Adaptivity to channel conditions is provided through a joint source-channel coding (JSCC) approach which attempts to jointly optimize the source and channel coding rates together with the modulation parameters to obtain the maximum achievable end-to-end QoS for the prevailing channel conditions. In this work, we model the wireless links as slow-fading Rician channel where the channel conditions can be described in terms of the channel signal-to-noise ratio (SNR) and the ratio of specular-to-diffuse energy $\zeta^{2}$. The multiresolution modulation/coding scheme consists of binary rate-compatible punctured convolutional (RCPC) codes used together with nonuniform phase-shift keyed (PSK) signaling constellations. Results indicate that this adaptive JSCC scheme employing scalable video encoding together with a multiresolution modulation/coding approach leads to significant improvements in delivered video quality for specified channel conditions. In particular, the approach results in considerably improved graceful degradation properties for decreasing channel SNR.
\end{abstract}

Copyright (C) 2007 Y. Pei and J. W. Modestino. This is an open access article distributed under the Creative Commons Attribution License, which permits unrestricted use, distribution, and reproduction in any medium, provided the original work is properly cited.

\section{INTRODUCTION}

The wireless channel varies over time and space and has short-term (or small-scale) memory due to multipath. These variations are caused either due to motion of the wireless device, or due to changes in the surrounding physical environment, and lead to detector errors. In addition to small-scale channel variations, there is also spatio-temporal variations on a much greater time scale [1]. Large-scale channel variation means that the average channel state condition depends on user locations and interference levels. As a result, it is wellrecognized now that cross-layer design is critically needed to insure continuity, robustness, and good end-to-end performance in multimedia wireless networks in the face of these random variations [2-8].

Most of the current explicit cross-layer design approaches have been limited to joint design between two layers [310]. Previous work $[9,11]$ described joint source-channel coding (JSCC) approaches for digital video transport over wireless links employing either a single-layer source coder with FEC or a 2-layer source coder in conjunction with FEC/UEP across layers to combat channel errors. Results indicate that with appropriate JSCC, tailored to the respective layers, FEC-based error control in combination with 2layer video coding techniques can lead to more acceptable quality for wireless video delivery in the presence of channel impairments. Specifically, in [11] the source and channel coded video data streams from different prioritized layers are multiplexed, and then modulated using uniform binary phase-shift keyed (BPSK) modulation before being transported over a wireless channel. This means that the data-link layer provides the same QoS for different prioritized layers, and UEP is achieved only through the use of different channel codes for the different prioritized layers.

Multiresolution modulation schemes, however, are capable of directly providing different QoS for different prioritized layers by mapping them into different layers in the signaling constellation. When used in conjunction with a 


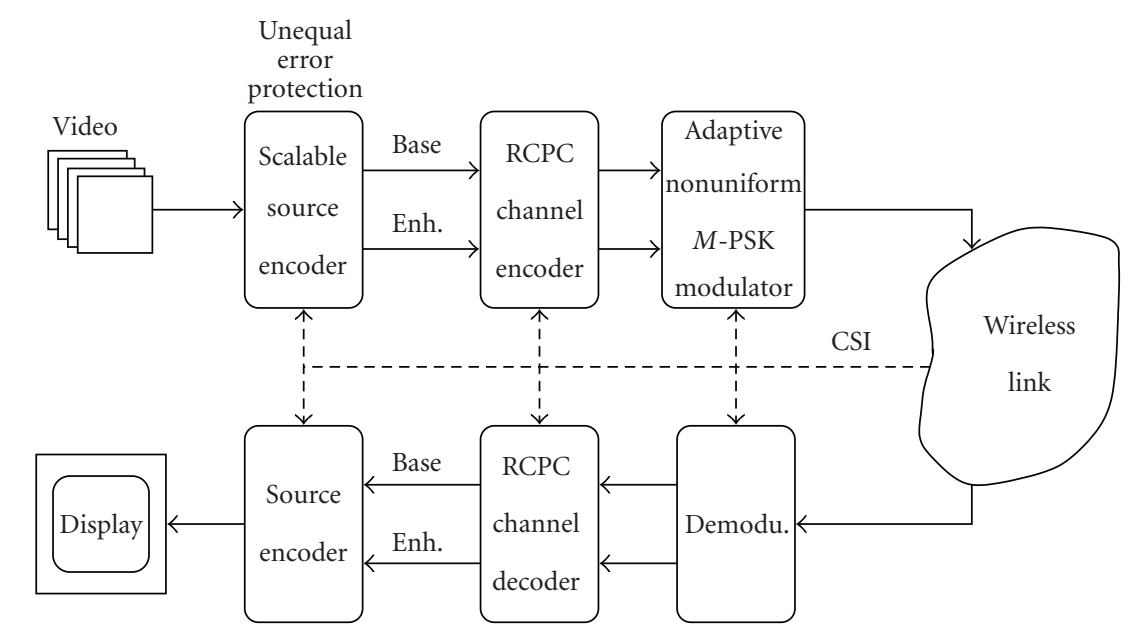

FIGURE 1: Illustration of a multilayered video coding and wireless delivery system.

FEC/UEP channel coding approach across layers, this leads to a more flexible and efficient JSCC procedure which is better able to exploit the differential sensitivities of the different source-encoded layers. Furthermore, such schemes can be used in an adaptive fashion by modifying the source coding rate as well as the channel modulation/coding strategy, based on the prevailing channel conditions, in an effort to maximize the end-to-end quality of the delivered video. Fixed transmission methods that are designed to provide the required QoS when channel conditions are poor are very inefficient when improved channel conditions prevail. Adaptation of the channel modulation/coding parameters permits maximum utilization of the wireless links in such systems as argued in [12]. Typically, these multiresolution modulation schemes adapt the size and/or shape of the signaling constellation as a function of the prevailing channel conditions. For example, when the channel conditions are good it is possible to use a higher-order signaling alphabet with less powerful FEC coding. This allows larger throughput which can support the transport of additional enhancement layers to improve the quality of the reconstructed video. Otherwise, when the channel conditions are poor, smaller signaling alphabets must be used together with more powerful FEC coding. The reduced throughput is then capable of supporting only the base layer with correspondingly lower reconstructed video quality. In this work, we extend the approach in [11] to an adaptive multiresolution modulation and coding scheme which combines a multilayer video encoding and delivery scheme with an adaptive nonuniform phase-shift keyed (PSK) modulation/coding strategy.

The remainder of this paper is organized as follows: in Section 2 we provide some technical preliminaries describing the source coding, multiresolution modulation scheme, the use of binary rate-compatible punctured convolutional (RCPC) codes, and passive error concealment for video. In Section 3, we briefly describe the channel models used and provide the performance analysis for the coded and uncoded systems employing nonuniform MPSK over Rician slow-fading channels. In Section 3, we provide a description of the JSCC methodology. In Section 4 the proposed adaptive multiresolution modulation and coding (AMCJSCC) scheme is discussed. In Section 5 we provide some selected experimental results together with a discussion. Finally, Section 6 provides a summary and conclusions.

\section{PRELIMINARIES}

In this paper, we describe and investigate an adaptive wireless video coding and delivery system which combines a scalable video codec with UEP across layers achieved through a combination of FEC and use of multiresolution modulation schemes using nonuniform MPSK signal constellations. Considering the typical bandwidth limitations of wireless channels, QCIF-format $(176 \times 144)$ video sequences are used in this work.

Figure 1 illustrates the video coding and wireless delivery scheme proposed and investigated in this paper. In this work, a 2-layer H.263+ coder [13] with signal-to-noise (SNR) scalability originally developed by the University of British Columbia and Telenor Group $[14,15]$ is used. The scalable H.263+ source coder encodes the input video into two layers, a base layer (Base) carrying the most important information and an enhancement layer (Enh) carrying the less important video information which, in turn, provides two VBR video streams with different priorities. The differential importance of encoder output components from different layers to the reconstructed video quality will be illustrated in what follows, and the results are used as the basis for the proposed prioritized video delivery scheme. The same scalable H.263+ source coder can also be used as a singlelayer VBR H.263+ coder together with a single-layer JSCC delivery scheme. This optimized single-layer system will be used as a baseline for comparison purposes.

For the 2-layer system, before the layers are transmitted, they are protected against channel errors according to their relative importance. A set of binary RCPC codes are 
employed on both layers for forward error correction. The channel coding rates can also be selected adaptively for both the base and enhancement layers based on the channel conditions. Then, the two video streams are modulated by using nonuniform MPSK signal constellations where the data from the base layer are mapped to the coarse resolution layer of the signaling constellation while the data from the enhancement layer are mapped to the finer resolution layer of the signaling constellation. Finally, the modulated signals are transmitted over a wireless link. During transmission, the modulated bitstreams typically undergo degradation due to AWGN, cochannel and/or inter-channel interference and possibly fading, specifically in this paper we model the channel as Rician slow-fading channel. At the receiver side, the received waveforms are demodulated and channel decoded, and then source decoded to form the reconstructed video sequence. The reconstructed sequence may differ from the original sequence due to both source coding errors and possible channel error effects.

\subsection{Performance analysis for RCPC codes over slow-fading Rician channel}

The class of FEC codes employed in this work is the set of binary rate-compatible punctured convolutional (RCPC) codes described in [16]. By deleting, or puncturing, bits from the coded bitstream, higher-rate codes are produced from lower-rate codes. The puncturing is controlled by a puncturing table which indicates which of the coded bits are to be transmitted and which are punctured.

The class of RCPC codes is especially well suited for a multilayered and/or adaptive transmission schemes, as the different priority classes may be provided different levels of protection, or UEP. By using a family of RCPC codes, these different levels of protection may be obtained from a given mother code using different puncturing tables. Furthermore, by switching between puncturing tables the levels of channel protection may be easily adapted to suit channel conditions for time-varying channels with a minimal number of coders as well as reduced decoder complexity.

An upper bound on the average symbol error probability is obtained as

$$
P_{c} \leq \frac{1}{P} \sum \sum_{\mathbf{x}, \hat{\mathbf{x}} \in C} a(\mathbf{x}, \widehat{\mathbf{x}}) p(\mathbf{x}) P(\mathbf{x} \longrightarrow \hat{\mathbf{x}}),
$$

where $a(\mathbf{x}, \hat{\mathbf{x}})$ is the number of symbol errors that occur when the sequence $\mathbf{x}$ is transmitted and the sequence $\hat{\mathbf{x}} \neq \mathbf{x}$ is chosen by the decoder, $p(\mathbf{x})$ is the a priori probability of transmitting $\mathbf{x}, C$ is the set of all coded sequences. Also in (2), $P(\mathbf{x} \rightarrow \widehat{\mathbf{x}})$ represents the pairwise error probability, that is, the probability that the decoder chooses $\widehat{\mathbf{x}}$ when indeed $\mathbf{x}$ was transmitted. $P$ is the puncturing period of the RCPC codes. The bit error probability can then be given as

$$
P_{b} \leq \frac{1}{P} \sum \sum_{\mathbf{x}, \hat{\mathbf{x}} \in C} c(\mathbf{x}, \hat{\mathbf{x}}) p(\mathbf{x}) P(\mathbf{x} \longrightarrow \hat{\mathbf{x}})
$$

where $c(\mathbf{x}, \hat{\mathbf{x}})$ is the corresponding number of bit errors that occur when the sequence $\mathbf{x}$ is transmitted and the sequence $\widehat{\mathbf{x}} \neq \mathbf{x}$ is chosen by the decoder.

The upper bounds (1) and (2) can be efficiently evaluated using the transfer-function bound approach [17]. Here, assuming ideal interleaving/deinterleaving, we consider the two extreme cases of channel state information (CSI): perfect CSI and no CSI. From the results in [17] we have

$$
P(\mathbf{x} \rightarrow \hat{\mathbf{x}}) \leq \exp \left\{-\frac{E_{s}}{4 N_{0}} d^{2}(\mathbf{x}, \hat{\mathbf{x}})\right\},
$$

where the "distance" metric is given by

$$
d^{2}(\mathbf{x}, \widehat{\mathbf{x}})= \begin{cases}d_{M E}^{2}(\mathbf{x}, \widehat{\mathbf{x}}) & \text { for perfect CSI } \\ d^{\prime 2}{ }_{M E}(\mathbf{x}, \widehat{\mathbf{x}}) & \text { for no CSI }\end{cases}
$$

The quantities $d_{M E}^{2}(\mathbf{x}, \widehat{\mathbf{x}})$ and ${d^{\prime 2}}_{M E}^{2}(\mathbf{x}, \widehat{\mathbf{x}})$ are the corresponding modified squared Euclidean distances as described below.

The symbol metric used to determine the coded system performance on the AWGN channel is the normalized Euclidean metric (or the squared Euclidean distance) which for MPSK signaling is given as

$$
d_{E}^{2}\left(\mathbf{x}_{i}, \widehat{\mathbf{x}}_{i}\right)=4 \sin ^{2} \frac{\pi\left(\mathbf{x}_{i}, \hat{\mathbf{x}}_{i}\right)}{M} ; \quad i=0, \pm 1, \pm 2, \ldots, \pm N
$$

However, as shown in $[17,18]$ the appropriate distance metric for fading channels must be modified to incorporate the fading effects. In particular, the appropriate symbol metric for a Rician channel with ideal interleaving/deinterleaving and perfect CSI is the normalized modified squared Euclidean metric given as [17]

$$
\begin{aligned}
d_{M E}^{2}\left(\mathbf{x}_{i}, \hat{\mathbf{x}}_{i}\right)= & \frac{\zeta^{2} d_{E}^{2}\left(\mathbf{x}_{i}, \hat{\mathbf{x}}_{i}\right)}{1+\zeta^{2}+\left(E_{s} / 4 N_{0}\right) d_{E}^{2}\left(\mathbf{x}_{i}, \hat{\mathbf{x}}_{i}\right)} \\
& +\left(\frac{E_{s}}{4 N_{0}}\right)^{-1} \ln \frac{1+\zeta^{2}\left(E_{s} / 4 N_{0}\right) d_{E}^{2}\left(\mathbf{x}_{i}, \hat{\mathbf{x}}_{i}\right)}{1+\zeta^{2}}
\end{aligned}
$$

whereas for the case of no CSI the corresponding normalized modified squared Euclidean metric is given as [17]

$$
d_{M E}^{\prime 2}\left(\mathbf{x}_{i}, \widehat{\mathbf{x}}_{i}\right)=\frac{\zeta^{2} d_{E}^{2}\left(\mathbf{x}_{i}, \widehat{\mathbf{x}}_{i}\right)}{1+\zeta^{2}+E_{s} / N_{0}} .
$$

\subsection{Nonuniform MPSK modulation}

In this work, we employ a similar multiresolution modulation scheme as the nonuniform MPSK modulation schemes used in [19] to increase the throughput of a packet-switched CDMA system. In what follows we restrict attention to $M=8$ although the approach is applicable to arbitrary $M=2^{m}, m>1$. The source-and channel-encoded baselayer video stream is modulated onto a carrier using Graycoded quadriphase-shift keyed (QPSK) modulation. Every two binary symbols are mapped into one QPSK symbol, as illustrated in Figure 2(a). The QPSK signaling constellation is converted to a nonuniform 8-PSK signal constellation 


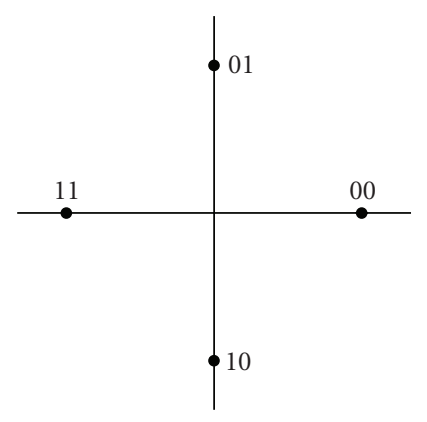

(a) QPSK

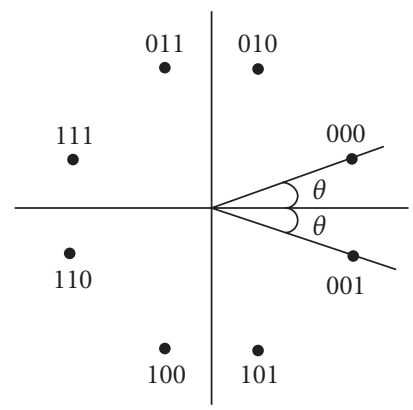

(b) Nonuniform 8-PSK

Figure 2: Adaptive nonuniform 8-PSK signaling constellation.

by splitting each point in the QPSK constellation into two points, each of which is rotated away from the original QPSK point by an angle $\theta$, as illustrated in Figure 2(b). The result is a nonuniform 8-PSK signal constellation with signals at angles $\theta,-\theta, \pi / 2+\theta, \pi / 2-\theta, \pi+\theta, \pi-\theta,-\pi / 2+\theta,-\pi / 2-\theta$. The base-layer data are represented by the pairs of binary symbols that appear as labels on the points in the constellation of Figure 2(a). The base-layer data also appear as the first two bits of the labels of the points in the 8-PSK constellation of Figure 2(b). The third bit of each label in Figure 2(b) is derived from the enhancement-layer data. The relative probabilities of error for the two message streams are controlled by varying the modulation parameter $\theta$, which is referred to as the offset angle in [19].

Considering that the base-layer data are of higher priority and require better protection, as demonstrated in [11], we allow the parameter $\theta$ to vary from 0 to $\pi / 8$, while in [19] $\theta$ can vary from 0 to $\pi / 4$ provided that the bit-error probability requirement for the base layer is satisfied.

Symbol error probability bounds are used to obtain the corresponding bit-error probabilities for data mapping to different resolutions of the signaling constellation. Firstly, as in [20], we model the sum of the interference and noise as stationary Gaussian noise with one-sided spectral density $N_{I}$, which represents the one-sided power spectral density for the interference and noise. If $E_{S}$ is the received energy per PSK symbol, then $E_{S} / N_{I}$ determines the corresponding symbolerror probability. In [12], Pursley and Shea derive error bounds for the nonuniform 8-PSK signaling constellation of
Figure 2(b); the error bounds for uniform QPSK and 8-PSK constellation are special cases with $\theta=0$ and $\pi / 8$, respectively. We make use of these same bounds in our work to evaluate the error probability for the base layer and the enhancement layer.

First we consider the system without channel coding. The bit-error probability for the base layer (i.e., the encoder output component sent using the coarse modulation of the nonuniform MPSK constellation) is approximated by $[12,19]$

$$
P_{b}^{(1)}(\theta) \approx \frac{1}{m-1}\left\{\frac{M-2}{M}-C\left(\frac{2 \pi}{M}-\theta\right)-C\left(\frac{2 \pi}{M}+\theta\right)\right\},
$$

where $C(\theta)$ is given by ${ }^{1}$

$$
\begin{aligned}
C(\theta)= & \frac{1}{4}\left\{1-2 Q\left(\sqrt{2} e_{s} \sin (\theta)\right)\right\} \\
& +\frac{1}{\sqrt{\pi}} \int_{0}^{e_{S} \sin \theta} \exp \left(-y^{2}\right)\left[\frac{1}{2}-Q(\sqrt{2} y \cot \theta)\right] d y .
\end{aligned}
$$

In (9), $e_{S}=\sqrt{E_{S} / N_{I}}$, where $E_{S}$ is the received energy per PSK symbol, and $N_{I} / 2$ is the two-sided power spectral density of the stationary AWGN as described above. We consider signaling alphabets with $M=2^{m}$, for example, for 8-PSK, $M=8$, and $m=3$.

For the information in the enhancement layer (i.e., the encoder output component sent using the fine modulation of the nonuniform MPSK constellation) an upper bound for the probability of bit error is given by [19]

$$
P_{b}^{(2)}(\theta) \leq \frac{1}{2}-C\left(\frac{4 \pi}{M}-\theta\right)-C(\theta) .
$$

For a fixed value of $E_{S} / N_{I}$, the probability of bit error for the base layer increases while the probability of bit error for the enhancement layer decreases as the offset angle $\theta$ is increased from 0 to $\pi / 8$. For each value of $E_{S} / N_{I}$, the optimum value of the offset angle is the value of $\theta$ for which the best quality of video, measured as the end-to-end distortion, is achieved. This gives the optimum choice of $\theta$ as a function of $E_{S} / N_{I}$.

In the system described in this work, RCPC codes are employed for both the base layer and the enhancement layer to combat channel errors. We assume the enhancement layer data are independent random variables with equal probability of 0 and 1 . Let $\wp$ denote the set of all trellis paths not generated by the all-zeros base-layer message sequence and let $E$ denote the event that there is an error made by the Viterbi decoder at a particular node of the decoding trellis assuming the all-zero sequence was transmitted. Let $p$ represent a specific trellis path $p \in \wp$ and let $n_{01}$ denote the number of base-layer message bit pairs of the form $(0,1)$,

\footnotetext{
${ }^{1}$ Here $Q(x)=1 / \sqrt{2 \pi} \int_{x}^{\infty} e^{-y^{2} / 2} d y$.
} 


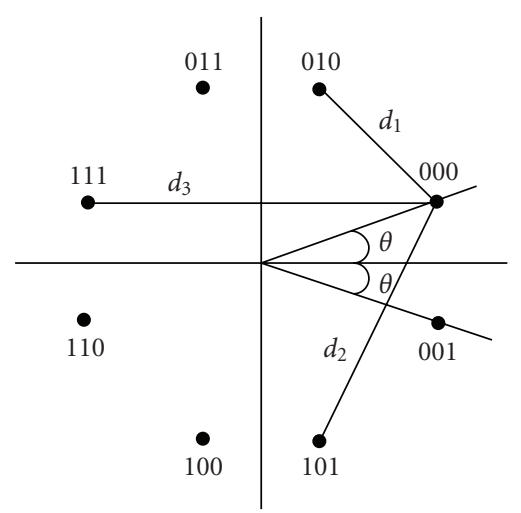

FIGURE 3: Euclidean distances for the nonuniform 8-PSK constellation.

let $n_{10}$ denote the number of base-layer message bit pairs of the form $(1,0)$, and let $n_{11}$ denote the number of baselayer message bit pairs of the form $(1,1)$. For a particular enhancement-layer sequence, let $\ell_{1}$ denote the number of symbols that represent the 01 base-layer message sequence for which the enhancement-layer message is 1 , and let $\ell_{2}$ denote the number of symbols that represent the 10 base-layer message sequence for which the enhancement-layer message is 1. The exact Euclidean distance between the all-zeros trellis path and a particular error path depends on the values of the enhancement-layer message bits and the mapping from the base-layer message pairs to 4 -ary symbols. Suppose a symbol that represents the base-layer message pair $(0,0)$ is transmitted. As illustrated in Figure 3, the squared distance to the closest of the symbols generated by the pairs $(0,1)$ or $(1,0)$ is $d_{1}^{2}=2(1-\sin (2 \theta))$, and the squared distance to the other symbols generated by the pairs $(0,1)$ or $(1,0)$ is $d_{2}^{2}=2$. The squared distance from the symbol representing $(0,0)$ to the closest symbol representing the encoded base-layer message pair $(1,1)$ is $d_{3}^{2}=4 \cos ^{2} \theta$. Then the number of symbols at distance $d_{1}$ is $n_{01}-\left(\ell_{1}-\ell_{2}\right)$, and the number of symbols at distance $d_{2}$ is $n_{10}+\left(\ell_{1}-\ell_{2}\right)$. Hence, the number of symbols at each distance depends on $\left(\ell_{1}-\ell_{2}\right)$ only. Let $\ell^{\prime}$ denote $\left(\ell_{1}-\ell_{2}\right)$. As given in $[12,16]$, the probability of event error for the base layer is bounded by

$P_{E}^{(1)} \leq \frac{1}{P} \sum_{p \in \wp}\left[\sum_{\ell^{\prime}=-n_{10}}^{n_{01}} Q\left(\frac{\|p\|_{\ell^{\prime}}}{\sqrt{2 N_{I}}}\right)\left(\begin{array}{c}n_{01}+n_{10} \\ \ell^{\prime}+n_{10}\end{array}\right)\left(\frac{1}{2}\right)^{n_{01}+n_{10}}\right]$,

where $P$ denotes the puncturing period of the RCPC codes, and $\|p\|_{\ell^{\prime}}^{2}$ is given by

$$
\|p\|_{\ell^{\prime}}^{2}=E_{S}\left[\left(n_{01}-\ell^{\prime}\right) d_{1}^{2}+\left(n_{10}+\ell^{\prime}\right) d_{2}^{2}+n_{11} d_{3}^{2}\right] .
$$

Letting $N_{p}$ denote the number of information bit errors resulting from the selection of an incorrect path $p \in \wp$, the corresponding bit-error probability is upper-bounded by

$P_{b}^{(1)} \leq \frac{1}{P} \sum_{p \in \wp} N_{p}\left[\sum_{\ell^{\prime}=-n_{10}}^{n_{01}} Q\left(\frac{\|p\|_{\ell^{\prime}}}{\sqrt{2 N_{I}}}\right)\left(\begin{array}{c}n_{01}+n_{10} \\ \ell^{\prime}+n_{10}\end{array}\right)\left(\frac{1}{2}\right)^{n_{01}+n_{10}}\right]$.
The probability of event error for the enhancement-layer message is bounded by [19]

$$
P_{E}^{(2)} \leq \frac{1}{P} \sum_{d=d_{\text {free }}}^{\infty} a(d) Q\left(\sqrt{\frac{d E_{s} \sin ^{2} \theta}{2 N_{I}}}\right)
$$

where $a(d)$ denotes the number of paths at Hamming distance $d$ from the all-zeros path and $d_{\text {free }}$ is the free distance of the code.

The probability of bit error for the enhancement-layer message is then bounded by

$$
P_{b}^{(2)} \leq \frac{1}{P} \sum_{d=d_{\text {free }}}^{\infty} c(d) Q\left(\sqrt{\frac{d E_{s} \sin ^{2} \theta}{2 N_{I}}}\right)
$$

where $c(d)$ denotes the total number of incorrectly decoded information bit errors for all the incorrect paths at Hamming distance $d$ from the all-zeros path.

\section{JOINT SOURCE-CHANNEL CODING METHODOLOGY}

The overall performance will be measured as the average PSNR over a sequence of $N_{f}$ consecutive frames and includes channel error effects as well as source coding losses. For a given modulation parameter $\theta$, assuming a $K$-layer system, ${ }^{2}$ PSNR $\left(\mathbf{R}_{s}, \mathbf{R}_{c}, \theta\right)$ can be determined for each combination of source coding rates, $\mathbf{R}_{s}=\left(R_{s}^{(1)}, R_{s}^{(2)}, \ldots, R_{s}^{(K)}\right)$, and channel coding rates, $\mathbf{R}_{c}=\left(R_{c}^{(1)}, R_{c}^{(2)}, \ldots, R_{c}^{(K)}\right)$, then the corresponding optimal operational distortion-rate characteristics for a given overall channel signaling rate $R_{s+c}$, in channel uses/source sample, is given as

$$
\operatorname{PSNR}^{*}\left(R_{s+c}, \theta\right)=\max \operatorname{PSNR}\left(\mathbf{R}_{s}, \mathbf{R}_{c}, \theta\right)
$$

where the maximization is performed over all $\mathbf{R}_{s}$ and $\mathbf{R}_{c}$ of interest, subject to the constraint

$$
\sum_{i=1}^{K} \frac{R_{s}^{(i)}}{R_{c}^{(i)}} \leq R_{s+c}
$$

Although we prefer to represent $R_{s+c}$ in normalized units, given the video format and frame rate it is relatively easy to convert ${ }^{3}$ to bits/second.

In $[21,22]$, it was shown that much of the computational complexity involved in solving this optimal rate allocation problem may be avoided through use of universal distortion-rate characteristics, $\operatorname{PSNR}\left(\mathbf{R}_{s}, \mathbf{P}_{b}\right)$, where $\mathbf{R}_{s}$ represents the source rate allocation vector for the various layers and $\mathbf{P}_{b}=\left(P_{b}^{(1)}, P_{b}^{(2)}, \ldots, P_{b}^{(K)}\right)$ represents the corresponding

\footnotetext{
2 The results in this paper are restricted to the 2-layer case with $K=2$.

${ }^{3}$ In particular, for the $4: 2: 0$ chrominance subsampling scheme used in H.263+ standard, the bit rate in bps is given by $r_{s+c}=(3 / 2)\left(N_{h} \times N_{v}\right) \times$ $f_{s} \times R_{s+c}$ with $f_{s}$ the frame rate.
} 


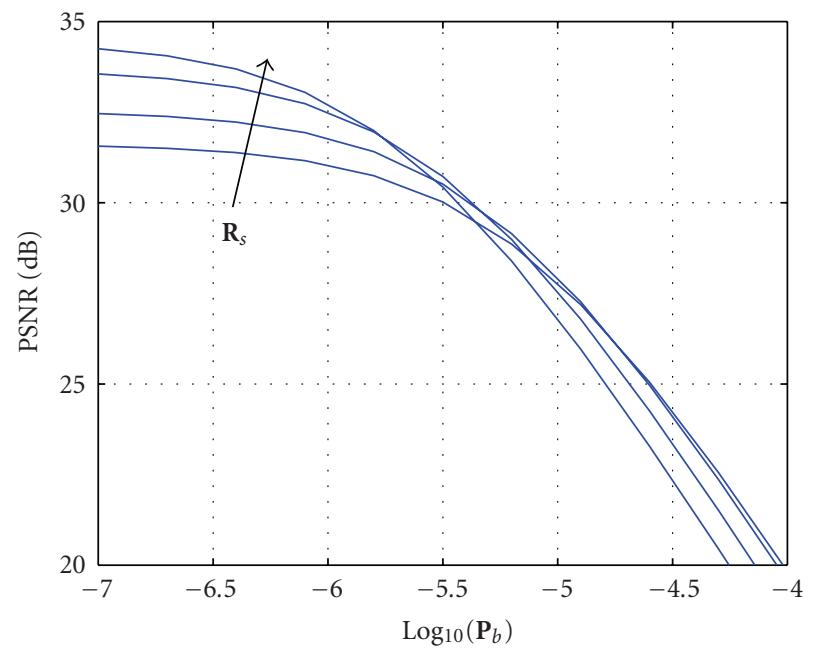

Figure 4: Typical universal rate-distortion characteristics for a single-layer H.263+ coder, PSNR $\left(\mathbf{R}_{s}, \mathbf{P}_{b}\right)$.

bit-error probabilities. For a single-layer H.263+ encoder, these are a family of curves $\operatorname{PSNR}\left(\mathbf{R}_{s}, \mathbf{P}_{b}\right)$ with specified source coding parameters indicating the PSNR as a function of the bit-error probability $P_{b}$ with $R_{s}$ as a parameter. Figure 4 shows a representative set of such curves obtained through simulation ${ }^{4}$ using $N_{f}=120$ frames of the QCIF Susie sequence at $f_{s}=30 \mathrm{fps}$. Observe, in particular, that for values of $P_{b}$ in excess of approximately $10^{-5}$ the PSNR is maximized for smaller values of $R_{s}$.

For the 2-layer H.263+ encoder, the overall distortion cannot be explicitly determined as the sum of the distortions of the base layer and enhancement layer, because the set of available rate-distortion operating points for the enhancement-layer codes depends on the particular choice of rate-distortion operating point for the baselayer codes. Hence, a trellis-based solution is required here as in [23]. As a result, the corresponding universal distortion-rate characteristics for a 2-layer coding scheme are families of surfaces with specified source coding parameters, PSNR $\left(R_{s}^{(1)}, R_{s}^{(2)}, P_{b}^{(1)}, P_{b}^{(2)}\right)$. Such a surface is shown in Figure 5, again for the Susie sequence, for the particular choice of $\mathrm{QPs}\left(\mathrm{QP}_{I}, \mathrm{QP}_{\mathrm{P}}, \mathrm{QP}_{\mathrm{Enh}}\right)=(2,6,3)$, corresponding to a fixed choice of $\mathbf{R}_{s}$. It clearly shows that the quality, measured in terms of PSNR, possesses different sensitivity to the bit-errors in the base-layer data and enhancement-layer data. In this case, the PSNR degrades much more dramatically due to the bit errors in the base layer than those in the enhancement layer. Intuitively, we can expect that employing UEP for the base and enhancement layers will be more efficient in the use of the limited bitrate.

In practice, computing the rate-distortion characteristics on the fly can be a challenge of applying JSCC approach.

\footnotetext{
${ }^{4}$ The curves for different $\mathbf{R}_{s}$ are obtained for fixed values of QPs.
}

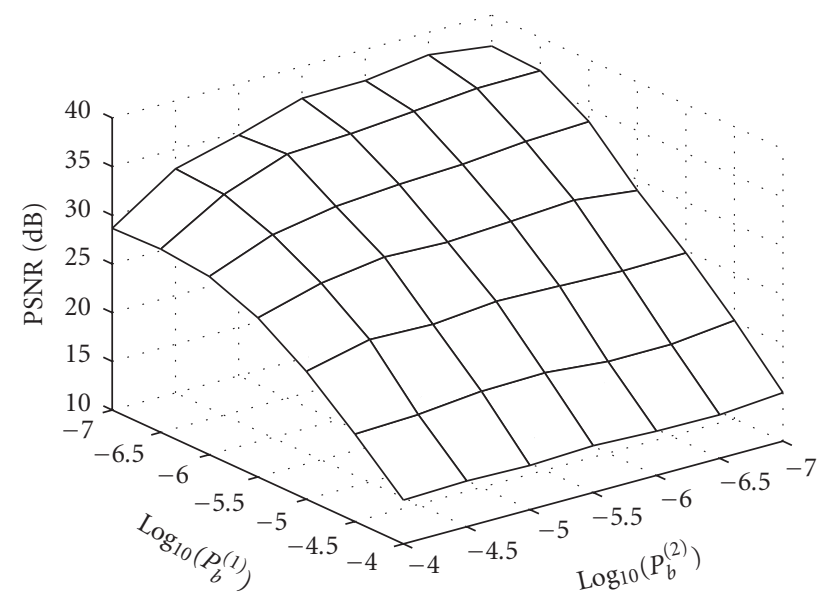

FIgURE 5: Typical universal rate-distortion characteristics $\operatorname{PSNR}\left(R_{s}^{(1)}, R_{s}^{(2)}, P_{b}^{(1)}, P_{b}^{(2)}\right)$ for a 2-layer SNR scalable H.263+ coder, for quantization parameters $\left(\mathrm{QP}_{I}, \mathrm{QP}_{P}, \mathrm{QP}_{\mathrm{Enh}}\right)=(2,6,3)$.

Many video applications belong to the video streaming category involving prestored video, while the other category is real-time interactive video application. For the video streaming applications, the required rate-distortion characteristics can be computed and stored in advance. For the real-time interactive video applications, videos can be classified into different representative classes, for example, based on the motion level; and the rate-distortion characteristics calculated from the representative video sequence of the corresponding class can be used for the JSCC adaptation even if directly calculating the precise rate-distortion characteristics for current video is not possible. In such a scenario, it may lead to a deduction in quality improvement from JSCC. Further study on this issue is worthwhile, but beyond the scope of this paper.

Given a family of universal distortion-rate characteristics for a specified source coder, together with appropriate bounds on bit-error probability for a particular modulation/coding scheme as a function of modulation and channel parameters, the corresponding optimal distortion-rate characteristics for a video sequence can be determined [21, 22] through the following procedure: for a specified channel signal-to-noise ratio, $E_{S} / N_{I}$, and modulation parameter, $\theta$, we can find the associated $\left(P_{b}^{(1)}(\theta), P_{b}^{(2)}(\theta)\right)$ through the corresponding bit-error probability bounds in (13) and (15) for a selected modulation/coding scheme as discussed earlier. ${ }^{5}$ Then, for each choice of source coding rate $\mathbf{R}_{s}=\left(R_{s}^{(1)}, R_{s}^{(2)}\right)$ of interest, use the resulting $\mathbf{P}_{b}=\left(P_{b}^{(1)}(\theta), P_{b}^{(2)}(\theta)\right)$ to find the corresponding overall PSNR from the universal distortionrate characteristics. Finally, we evaluate the resulting component distortion-rate characteristics through a JSCC approach

\footnotetext{
${ }^{5}$ In particular, this entails specification of the channel coding rate vector $\mathbf{R}_{c}=\left(R_{c}^{(1)}, R_{c}^{(2)}\right)$ for a specified class of channel codes.
} 


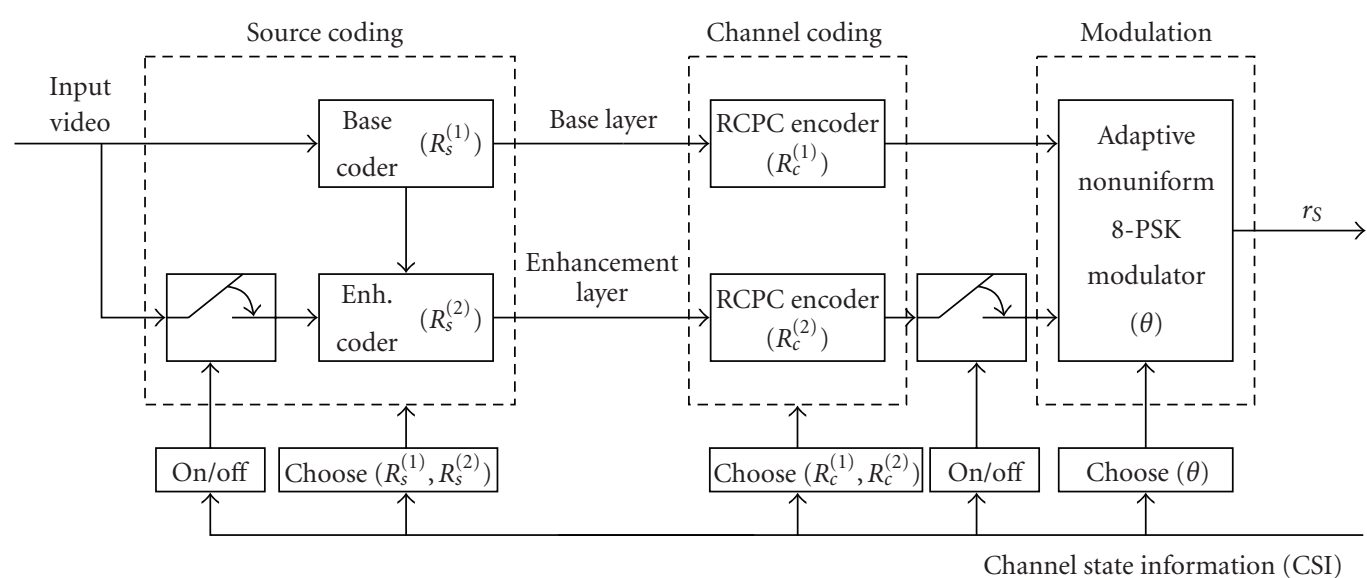

FIgURE 6: Adaptive multiresolution modulation and coding scheme for wireless delivery of digital video.

representing an extension of the single-layer procedure described in $[21,22]$. More specifically, this entails solution of the rate allocation problem described by (16) or, equivalently, obtaining the convex hull of all operational points $\operatorname{PSNR}\left(R_{s}^{(1)}, R_{s}^{(2)}, R_{c}^{(1)}, R_{c}^{(2)}, \theta\right)$ satisfying the constraint (17). In most of this work, $\left(R_{c}^{(1)}, R_{c}^{(2)}\right)$ are selected from a set of available RCPC codes of rates, $R_{c}=8 / 9,8 / 10, \ldots, 8 / 32$, which are obtained by making use of an $R_{c}=1 / 4$ mother code with memory $M=10$ and a corresponding puncturing period $P=8$.

\section{ADAPTIVE MULTIRESOLUTION MODULATION AND CODING SCHEME}

A block diagram of the proposed adaptive multiresolution modulation/coding (AMC) system is illustrated in Figure 6. The source encoder encodes the input video into either a single or dual streams. In either case, channel coding is provided by an RCPC channel encoder(s). The encoded messages are then mapped to the nonuniform 8-PSK signaling constellation as described in Section 2.2. As illustrated in Figure 6, adaptation is accomplished by adaptively adjusting the offset angle $\theta$, switching the encoder on or off for the enhancement layer, and choosing the values of the source and channel coding rates, $\mathbf{R}_{s}$ and $\mathbf{R}_{c}$, respectively, through JSCC subject to the overall transmission rate $R_{s+c}$, according to the channel state information (CSI). ${ }^{6}$ As the channel conditions change, these parameters are adapted to provide the best end-to-end quality of the delivered video, subject to the overall bit budget, which is given by

$$
\operatorname{PSNR}^{*}\left(R_{s+c}\right)=\max \operatorname{PSNR}\left(\mathbf{R}_{s}, \mathbf{R}_{c}, \theta\right),
$$

where the maximization is performed over all $\mathbf{R}_{s}, \mathbf{R}_{c}$, and $\theta$ of interest, subject to the constraint given in (17).

As discussed previously, we firstly model the sum of interference and noise as stationary AWGN with one-sided

\footnotetext{
${ }^{6}$ In the work described here, the CSI consists simply of knowledge of $E_{S} / N_{I}$.
}

spectral density $N_{I}$. If $E_{S}$ is the energy per symbol, then $E_{S} / N_{I}$ determines the error probability for both layers, that is, for a fixed value of $E_{S} / N_{I}$, the probability of error for the base layer increases as the offset angle $\theta$ is increased, while the probability of error for the enhancement layer decreases as the offset angle $\theta$ is increased. The constrained maximization over $\theta$ in (18) determines the optimum choice of $\theta$ as a function of $E_{S} / N_{I}$. If Rician fading channel model instead of AWGN channel model is used, $E_{S} / N_{I}$ together with $\zeta^{2}$ should be taken into consideration in the process to evaluate the probability of errors.

The adaptation process of this AMC-JSCC approach is as follows: consider the case in which the transmitter employs the proposed adaptive multiresolution modulation and coding scheme to send video to a remote receiver. We assume that CSI is available such that the transmitter can adapt the transmission parameters based on this knowledge. Once the transmitter knows the channel conditions, it will adjust all the parameters based upon the operational rate-distortion characteristics available at the transmitter side.

We include the ability of the adaptive scheme to be able to switch the source coder between a single-layer coding mode and a 2-layer coding mode. The motivation for this is based on the fact that, compared to a single-layer encoder, scalable coding schemes suffer relative performance degradations in the absence of channel errors primarily due to the additional overheads associated with the layered approach. This mode switching is accomplished, as indicated in Figure 6, by monitoring the optimized value of $\theta$. For example, whenever this value is equal to $\pi / 8$, corresponding to uniform 8 -PSK, we eliminate the enhancement layer by setting $R_{s}^{(2)}=0$ and use the output of the base layer to choose the 8-PSK symbol. The two switches in Figure 6 effectively eliminate the enhancement layer, thereby reverting to a single-layer system.

\section{RESULTS AND DISCUSSION}

We present some selected results for the following video coding and transport schemes for a representative QCIF videoconferencing sequence, Susie at $30 \mathrm{fps}$. 


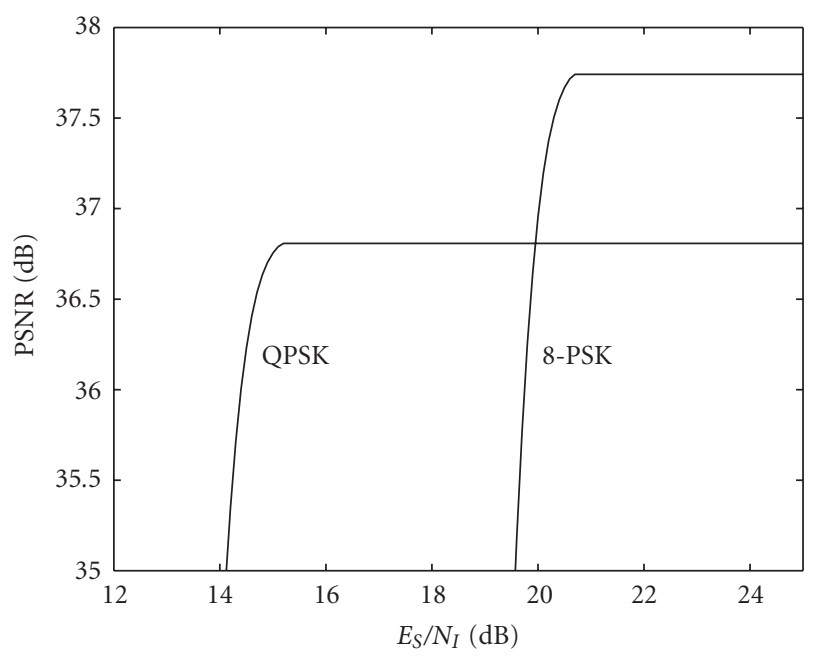

FIgURE 7: PSNR as a function of $E_{S} / N_{I}$ in $\mathrm{dB}$ for single-layer schemes employing uniform MPSK: QPSK and 8-PSK, without channel coding for AWGN channel. Fixed symbol transmission rate $r_{S}=128$ Ksps.

(1) A single-layer system using either uniform QPSK or uniform 8-PSK without channel coding.

(2) A 2-layer system using nonuniform 8-PSK without channel coding.

(3) A single-layer system using either uniform QPSK or uniform 8-PSK with channel coding.

(4) A 2-layer system using nonuniform 8-PSK with channel coding.

(5) The proposed adaptive 2-layer modulation/coding system using nonuniform 8-PSK and employing JSCC.

The symbol transmission rate is set to be $r_{S}=128 \mathrm{Ksps}$. For a single-layer system, if uniform QPSK is used as modulation, the message bitrate (after channel coding) is $r_{s+c}=$ $256 \mathrm{Kbps}$; if uniform 8-PSK is used as modulation, $r_{s+c}=$ $384 \mathrm{Kbps}$. For a 2-layer system employing nonuniform 8PSK modulation, the message bitrate (after channel coding) for the base layer is $r_{s+c}^{(1)}=256 \mathrm{Kbps}$, while for the enhancement layer $r_{s+c}^{(2)}=128 \mathrm{Kbps}$.

We first evaluate the performance of a single-layer system without channel coding and using uniform MPSK modulation for the AWGN channel. The results are demonstrated in Figure 7 for $M=4$ (QPSK) and $M=8$ (8-PSK). As expected, QPSK shows better performance in the range of lower $E_{S} / N_{I}$; however, as channel conditions improve (i.e., $E_{S} / N_{I}$ increases) the PSNR will saturate quickly for QPSK which makes the system very inefficient for large $E_{S} / N_{I}$. On the other hand, 8-PSK will provide better efficiency for large $E_{S} / N_{I}$ by allowing larger $r_{s+c}$, but at the expense of poorer performance as $E_{S} / N_{I}$ decreases compared to QPSK. Intuitively, a simple adaptive scheme could be devised to switch between the QPSK and 8-PSK based on the different values of $E_{S} / N_{I}$. This scheme will provide performance which is the upper envelope of the two curves shown in Figure 7.

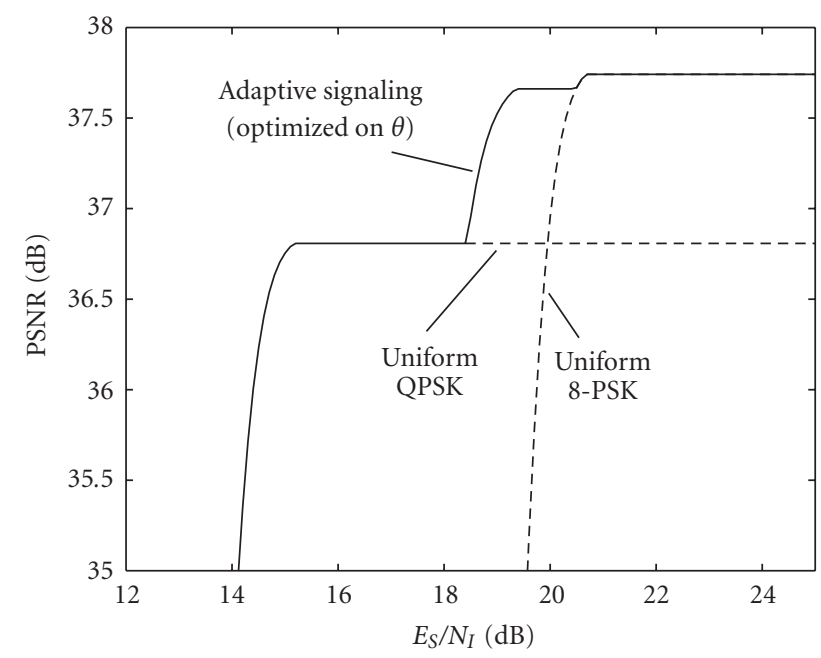

FIgURE 8: PSNR as a function of $E_{S} / N_{I}$ in $\mathrm{dB}$ for 2-layer system with adaptive modulation scheme without channel coding for AWGN channel. Fixed symbol transmission rate $r_{S}=128$ Ksps.

Instead, if adaptive nonuniform 8-PSK modulation is employed combined with a 2-layer source coding scheme for the uncoded system, we expect to get improved performance in the transition region between QPSK and 8-PSK for an uncoded system. The results are demonstrated in Figure 8. As can be seen, the adaptive 2-layer nonuniform 8-PSK modulation scheme demonstrates an advantage in keeping the performance at acceptable levels for the lower $E_{S} / N_{I}$ by reverting to a QPSK $(\theta=0)$ modulation scheme, then as $E_{S} / N_{I}$ increases to approximately $18.5 \mathrm{~dB}$, the enhancement-layer data can be used to improve the performance. Further increase in $E_{S} / N_{I}$ causes the optimum value of $\theta$ to increase resulting in a decrease in the bit error rate for the enhancement layer. As $E_{S} / N_{I}$ becomes large enough, the performance saturates at a level slightly below that of the single-layer system using uniform 8-PSK $(\theta=\pi / 8)$ at large $E_{S} / N_{I}$. This gap is the penalty to be paid for 2-layer scalable source coding compared to single-layer source coding. In particular, this performance gap is why we provide a switch in the adaptive modulation/coding scheme to revert to a single-layer source coding scheme for large $E_{S} / N_{I}$. Then as $E_{S} / N_{I}$ becomes large enough, the adaptive nonuniform 8-PSK modulation scheme reverts to a conventional uniform 8 -PSK $(\theta=\pi / 8)$ modulation scheme supporting a single-layer encoder. So we see that by adjusting $\theta$ adaptively, it provides a more graceful degradation pattern compared to the single-layer system employing uniform modulation schemes. This indicates that if CSI is available to the transmitter, the 2-layer encoding scheme with adaptive nonuniform modulation can be used to obtain a considerable performance improvement in the quality of the delivered video.

Similar features are obtained for the Rician fading channel as demonstrated in Figure 9, where we consider a Rician fading channel with $\zeta^{2}=7 \mathrm{~dB}$. We see that by adjusting $\theta$ adaptively, it provides a much more graceful degradation 
pattern compared to the single-layer system employing uniform modulation schemes.

In addition to the adaptive modulation, FEC can be used to protect the video data against channel errors to further improve the video delivery performance in the range of lower $E_{S} / N_{I}$ as demonstrated in $[10,11]$. Here, we will illustrate this through a specific case. We apply a code with $R_{c}=1 / 2$ from the set of RCPC codes to the single-layer encoded video stream with uniform modulation; for the 2-layer system, the same $R_{c}^{(1)}=1 / 2$ code is used for the base layer and an RCPC code with $R_{c}^{(2)}=1 / 3$ is used for the enhancement layer. ${ }^{7}$ The results are demonstrated in Figure 10 for AWGN channel. For lower values of $E_{S} / N_{I}$ (e.g., $E_{S} / N_{I} \leq 7 \mathrm{~dB}$ ), as the adaptive modulation scheme reverts to a single-layer uniform QPSK scheme, the 2-layer system performs essentially the same as the single-layer system using uniform QPSK. As a result, in Figure 10 the corresponding two curves overlap in this area. On the other hand, for larger values of $E_{S} / N_{I}$ (e.g., $E_{S} / N_{I} \geq 10 \mathrm{~dB}$ ), as the adaptive scheme reverts to uniform 8-PSK, the 2-layer system performs essentially the same as a single-layer system using uniform 8-PSK. However, in the intermediate transition range, corresponding to intermediate values of $E_{S} / N_{I}$, demonstrates a decided advantage and provides a more graceful performance degradation pattern by adaptively adjusting the modulation parameter, that is, the offset angle $\theta$. Again, this graceful degradation property allows the performance to be maintained at acceptable levels for lower values of $E_{S} / N_{I}$ while simultaneously improving the performance gracefully as $E_{S} / N_{I}$ increases. Compared to the results in Figure 8, the use of FEC can be seen to significantly improve the performance compared to the case without channel coding for lower $E_{S} / N_{I}$, while suffering some quality loss for large $E_{S} / N_{I}$ due to the channel coding overhead. This suggests that FEC is necessary for wireless video delivery to achieve acceptable quality for the small values of $E_{S} / N_{I}$ of interest. ${ }^{8}$ On the other hand, the channel codes must be carefully selected, otherwise the coded system will be inefficient for larger $E_{S} / N_{I}$. Adaptive scheme demonstrates the graceful degradation property of keeping the performance at acceptable level for lower values of $E_{S} / N_{I}$ while simultaneously improving the performance gracefully as $E_{S} / N_{I}$ increases. It should be noted that these results were for a quite arbitrary choice of channel codes and no attempt was made to select these rates to optimize the end-to-end performance as in a JSCC approach.

The works in $[10,11]$ demonstrated the advantages of using JSCC to improve the overall performance of video delivery. In this work, we further investigate the performance of our proposed adaptive 2-layer modulation/coding scheme

\footnotetext{
7 Typically, for a uniform MPSK signaling scheme, we would expect $R_{c}^{(1)} \leq$ $R_{c}^{(2)}$ to optimize the performance. However, for the adaptive nonuniform modulation/coding scheme considered here, this is no longer the case since unequal error protection is provided through both the nonuniform modulation and channel coding. As a result, this choice is not unreasonable.

${ }^{8}$ Unless $E_{S} / N_{I}$ is kept small, the multiple-access interference levels become excessively high, thereby reducing overall system capacity.
}

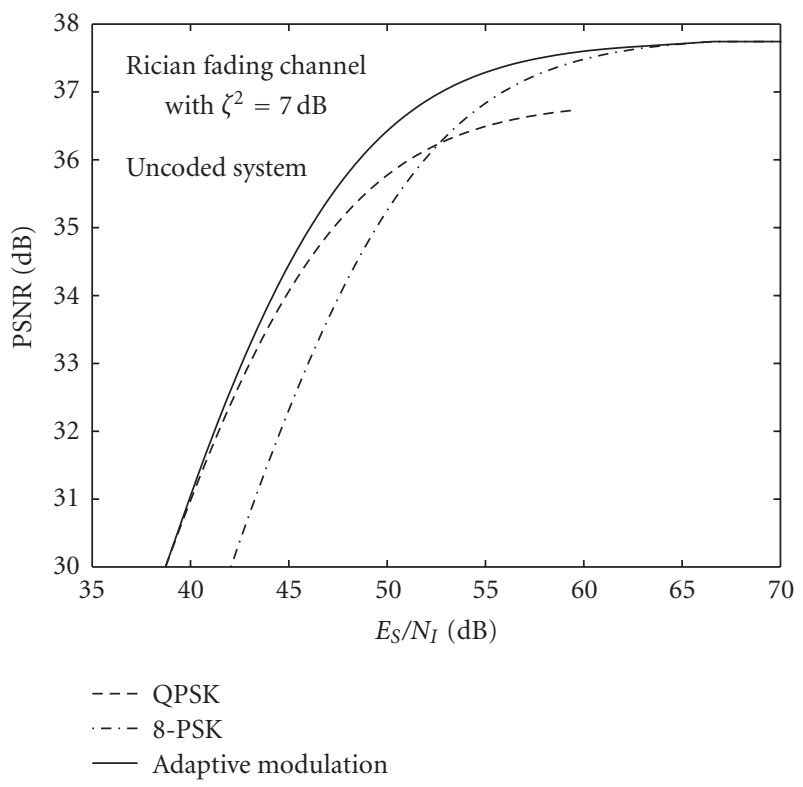

FIgURe 9: PSNR as a function of $E_{S} / N_{I}$ in $\mathrm{dB}$ for 2-layer system with adaptive modulation scheme without channel coding for Rician fading channel with $\zeta^{2}=7 \mathrm{~dB}$. Fixed symbol transmission rate $r_{S}=128$ Ksps.

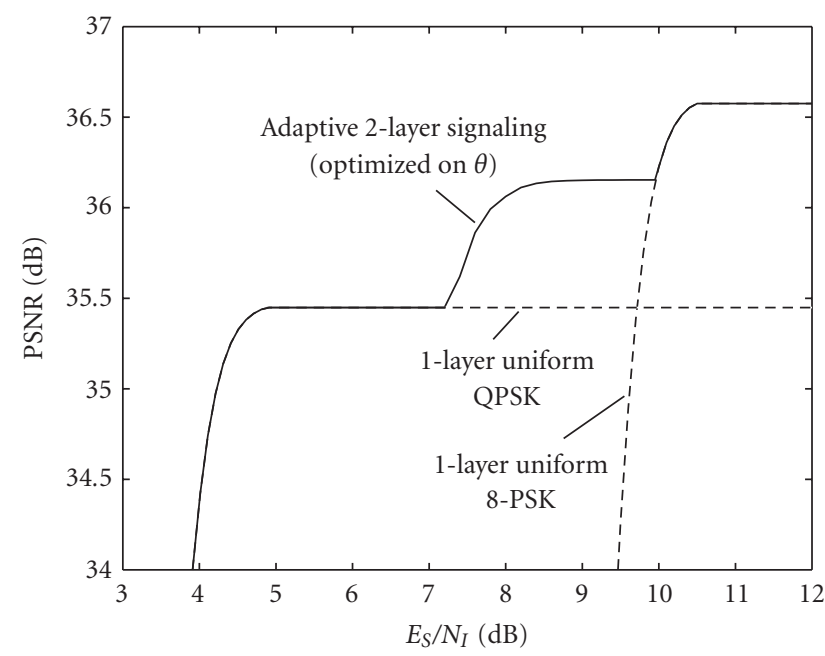

FIGURE 10: PSNR as a function of $E_{S} / N_{I}$ in $\mathrm{dB}$ for AWGN channel: (1) 1-layer schemes with fixed channel code using uniform QPSK or 8-PSK, and (2) a 2-layer adaptive modulation scheme with fixed channel codes, optimized on $\theta$. Fixed symbol transmission rate $r_{S}=$ 128 Ksps.

employing JSCC compared to those using only single-layer coding and uniform MPSK either with or without JSCC. The results are demonstrated in Figures 11 and 12 for the AWGN and Rician fading channels, respectively. For the AWGN channel, we see that for lower values of $E_{S} / N_{I}$ (e.g., $E_{S} / N_{I} \leq$ $8 \mathrm{~dB}$ ), the adaptive scheme performs essentially the same as single-layer coding with JSCC and uniform QPSK. On the 


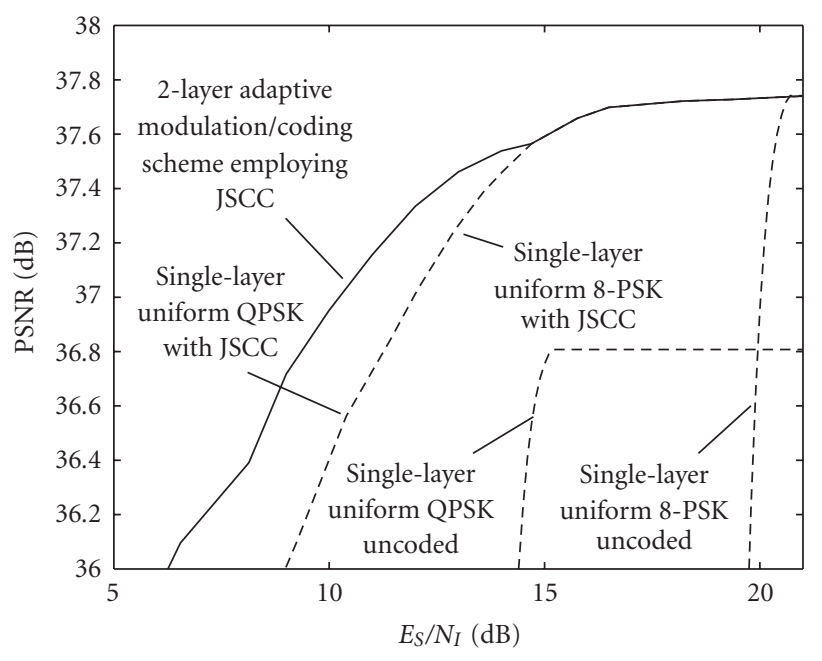

FIgURE 11: PSNR as a function of $E_{S} / N_{I}$ in $\mathrm{dB}$ for 2-layer adaptive modulation and coding scheme for AWGN channel. Fixed symbol transmission rate $r_{S}=128 \mathrm{Ksps}$.

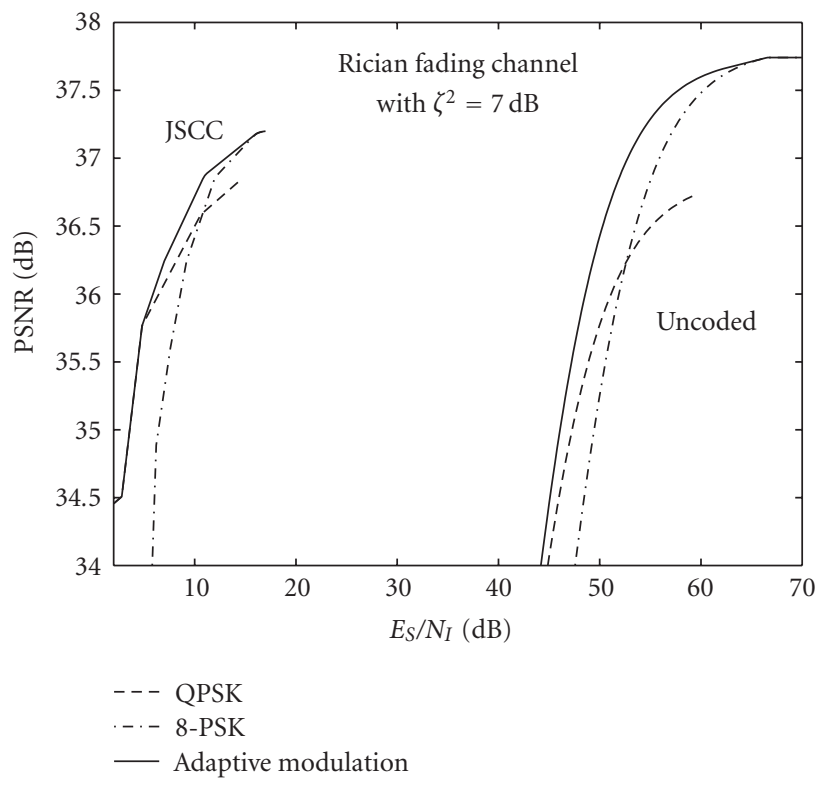

FIgURE 12: PSNR as a function of $E_{S} / N_{I}$ in $\mathrm{dB}$ for 2-layer adaptive modulation and coding scheme for Rician fading channel with $\zeta^{2}=$ $7 \mathrm{~dB}$. Fixed symbol transmission rate $r_{S}=128 \mathrm{Ksps}$.

other hand, for larger values of $E_{S} / N_{I}$ (e.g., $E_{S} / N_{I} \geq 15 \mathrm{~dB}$ ), the adaptive scheme performs essentially the same as singlelayer coding with JSCC and uniform 8-PSK. However, in the intermediate transition range (e.g., $8 \mathrm{~dB}<E_{S} / N_{I}<15 \mathrm{~dB}$ ), the 2-layer adaptive scheme demonstrates a significant advantage and provides a much more graceful performance degradation pattern achieved by means of adaptively adjusting the modulation parameter $\theta$ together with the use of JSCC. Specifically, as shown in Figure 11 there is a gain of approximately $1.8 \mathrm{~dB}$ in $E_{S} / N_{I}$ for a fixed quality level PSNR $=37 \mathrm{~dB}$. This improvement in energy efficiency can lead to a significant improvement in overall system capacity.

Further objective as well as subjective results for the AMC-JSCC systems compared to uncoded systems with fixed modulation are presented. The typical reconstructed video quality for selected channel conditions are demonstrated in Figure 13. Figure 13 shows the 12th frame of Susie subsequence $(N=12)$ with overall rate held constant at $r_{S}=$ $128 \mathrm{Ksps}$ for the AMC-JSCC system over a Rician fading channel with $\zeta^{2}=7 \mathrm{~dB}$ for channel $E_{S} / N_{I}=2,5,10$, and $15 \mathrm{~dB}$. For comparison, we also present the results for an uncoded system employing fixed QPSK modulation over a Rician fading channel with $\zeta^{2}=7 \mathrm{~dB}$ for channel $E_{S} / N_{I}=$ $20,30,60$, and $70 \mathrm{~dB}$. It is clear that extremely large $E_{S} / N_{I}$, above $30 \mathrm{~dB}$, is required for uncoded system to achieve acceptable quality over the fading channel, resulting in extremely high interference to other users sharing the same network, which is prohibitive in a multiuser wireless communication system where efficient low-power operation is the key to improved system capacity. On the other hand, due to the fixed modulation scheme, further improvement in throughput cannot be obtained through solely increasing the transmitted power level, say $E_{S} / N_{I}>60 \mathrm{~dB}$, even when such high transmitted power is allowable, for example, when there is only a single user in the network.

Instead, the AMC-JSCC system can avoid such problems and achieve graceful quality adjustment through the use of adaptive coding and modulation according to prevailing channel conditions, resulting in substantially improved reconstructed video quality transmitted through the wireless links as demonstrated in Figure 13. In contrast to uncoded system, reconstructed video with gracefully degrading quality can be obtained for the fading channel with $E_{S} / N_{I}$ as low as $2 \mathrm{~dB}$. To obtain reconstructed video with a reasonably good quality, say $34 \mathrm{~dB}$, the corresponding $E_{S} / N_{I}$ required is only $5 \mathrm{~dB}$. This offers the potential of significant improvements in system capacity. Furthermore, as the number of users sharing the same network resources decreases, larger operating power level may be allowed. For an AMCJSCC system, it may exploit this additional resource available to improve the throughput by adjusting the modulation constellation size and/or corresponding modulation parameters as demonstrated by the above adaptive nonuniform 8PSK system. As a result, further improvement in video quality is still possible in such an AMC-JSCC system. Considering that mobile wireless network condition is highly timevarying while moving inside a single cell and/or roaming between different cells, such an adaptive feature is of significant advantage to end-user quality as well as system capacity.

\section{SUMMARY AND CONCLUSIONS}

We have described and investigated a wireless video coding and delivery system which combines a scalable video codec with unequal error protection (UEP) across layers through a combination of FEC and multiresolution modulation schemes using nonuniform MPSK signal constellations. The 


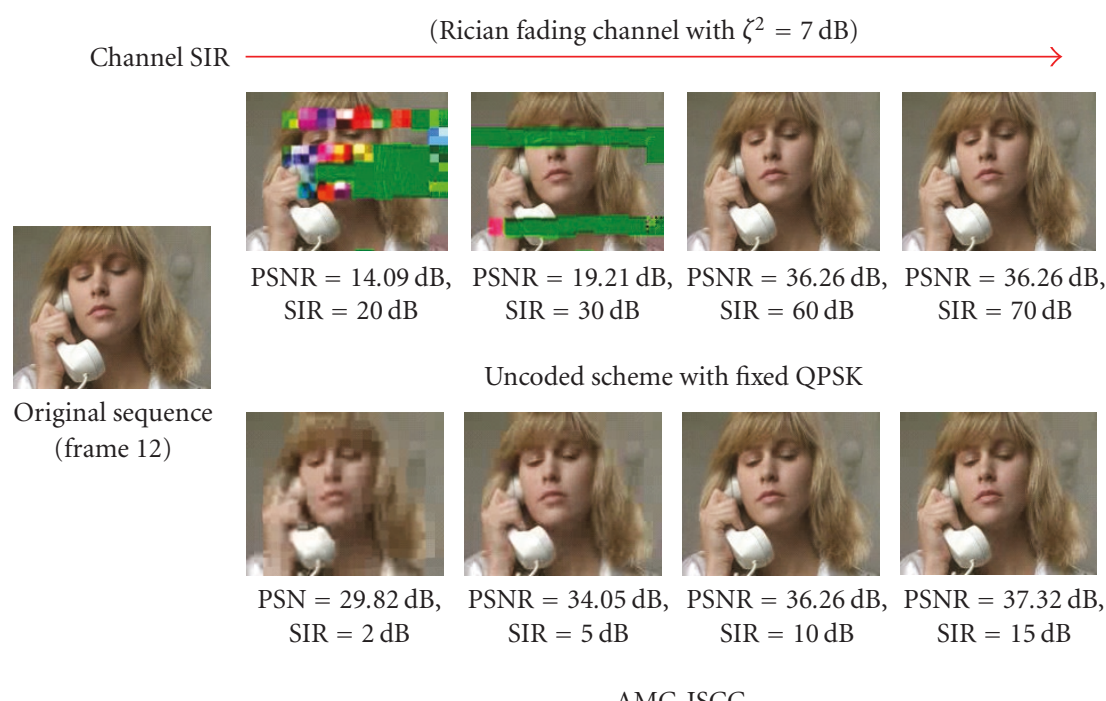

FIgURE 13: The 12th frame of Susie subsequence $(N=12)$ with overall rate held constant at $r_{S}=128$ Ksps for an AMC-JSCC system employing RCPC codes and adaptive nonuniform 8-PSK modulation over Rician fading channel with $\zeta^{2}=7 \mathrm{~dB}$.

results clearly demonstrate that FEC is required to maintain the video quality at an acceptable level for relatively small values of $E_{S} / N_{I}$. Furthermore, in order to maintain the video quality at acceptable levels over a relatively wide range of $E_{S} / N_{I}$ (i.e., the case for typical time-varying wireless links), JSCC is required to adaptively choose source and channel coding rates based on CSI, in order to protect the data against channel errors while operating within a fixed bandwidth allocation. Finally, adaptive modulation/coding schemes can be used to obtain improved performance for smaller $E_{S} / N_{I}$, while allowing higher throughput for larger $E_{S} / N_{I}$. More specifically, 2-layer adaptive modulation/coding schemes can provide much more graceful degradation characteristics between these two extreme ranges of $E_{S} / N_{I}$. Hence, multilayered video encoding and delivery with adaptive modulation/coding approaches, such as described here, should provide a significant system advantage for future wireless multimedia transmission systems.

\section{REFERENCES}

[1] T. S. Rappaport, Wireless Communications: Principles and Practice, Prentice Hall, Upper Saddle River, NJ, USA, 1996.

[2] Y. S. Chan and J. W. Modestino, "A joint source coding-power control approach for video transmission over CDMA networks," IEEE Journal on Selected Areas in Communications, vol. 21, no. 10, pp. 1516-1525, 2003.

[3] P. C. Cosman, J. K. Rogers, P. G. Sherwood, and K. Zeger, "Combined forward error control and packetized zerotree wavelet encoding for transmission of images over varying channels," IEEE Transactions on Image Processing, vol. 9, no. 6, pp. 982-993, 2000.

[4] Q.-F. Zhu and L. Kerofsky, "Joint source coding, transport processing, and error concealment for H.323-based packet video," in Visual Communications and Image Processing '99, vol. 3653 of Proceedings of SPIE, pp. 52-62, San Jose, Calif, USA, January 1999.

[5] P. Cherriman, C. H. Wong, and L. Hanzo, "Turbo- and BCHcoded wide-band burst-by-burst adaptive H.263-assisted wireless video telephony," IEEE Transactions on Circuits and Systems for Video Technology, vol. 10, no. 8, pp. 1355-1363, 2000.

[6] T. Chu and Z. Xiong, "Combined wavelet video coding and error control for internet streaming and multicast," Eurasip Journal on Applied Signal Processing, vol. 2003, no. 1, pp. 66-80, 2003.

[7] P. G. Sherwood, X. Tian, and K. Zeger, "Efficient image and channel coding for wireless packet networks," in Proceedigns of IEEE International Conference on Image Processing (ICIP '00), vol. 2, pp. 132-135, Vancouver, BC, Canada, September 2000.

[8] W. Kumwilaisak, J. W. Kim, and C.-C. J. Kuo, "Video transmission over wireless fading channels with adaptive FEC," in Proceedigns of the 22nd Picture Coding Symposium (PCS '01), pp. 219-222, Seoul, South Korea, April 2001.

[9] M. J. Ruf and J. W. Modestino, "Operational rate-distortion performance for joint source and channel coding of images," IEEE Transactions on Image Processing, vol. 8, no. 3, pp. 305320, 1999.

[10] M. Bystrom, J. W. Modestino, and Y. Pei, "Combined sourcechannel coding for wireless transmission of H.263 coded video," in Proceedigns of UCSD Conference on Wireless Communications, pp. 36-49, San Diego, Calif, USA, February 1999.

[11] L. P. Kondi, F. Ishtiaq, and A. K. Katsaggelos, "Joint sourcechannel coding for motion-compensated DCT-based SNR scalable video," IEEE Transactions on Image Processing, vol. 11, no. 9, pp. 1043-1052, 2002.

[12] M. B. Pursley and J. M. Shea, "Adaptive nonuniform phaseshift-key modulation for multimedia traffic in wireless networks," IEEE Journal on Selected Areas in Communications, vol. 18, no. 8, pp. 1394-1407, 2000.

[13] ITU-T/SG15, "Video Coding for Low Bitrate Communication," Draft Recommendation H.263 version 2, September 1997. 
[14] “H.263+Video Codec, Version 3.12," ECE Department, University of British Columbia, January 1998.

[15] Telenor Research, "Video Codec Test Model TMN5," January 1995, http://www.fou.telenor.no/ brukere/DVC/tmn5.

[16] J. Hagenauer, "Rate-compatible punctured convolutional codes (RCPC Codes) and their applications," IEEE Transactions on Communications, vol. 36, no. 4, pp. 389-400, 1988.

[17] K. Park, Digital modulation and coding design for wireless channels, Ph.D. thesis, ECSE Department, Rensselaer Polytechnic Institute, Troy, NY, USA, 1995.

[18] E. Biglieri, D. Divsalar, P. J. McLane, and M. K. Simon, Introduction to Trellis-Coded Modulation with Applications, Macmillan, New York, NY, USA, 1991.

[19] M. B. Pursley and J. M. Shea, "Adaptive signaling for multimedia transmission in CDMA cellular radio systems," in Proceedigns of IEEE Military Communications Conference (MILCOM '98), vol. 1, pp. 113-117, Bedford, Mass, USA, October 1998.

[20] A. J. Viterbi, CDMA: Principles of Spread Spectrum Communication, Addison-Wesley, Reading, Mass, USA, 1995.

[21] M. Bystrom and J. W. Modestino, "Combined source-channel coding for transmission of H.263 coded video with trelliscoded modulation over a slow-fading Rician channel," in Proceedings of IEEE International Symposium on Information Theory (ISIT '98), vol. 2, p. 12, Cambridge, Mass, USA, August 1998.

[22] M. Bystrom and J. W. Modestino, "Combined source-channel coding schemes for video transmission over an additive white Gaussian noise channel," IEEE Journal on Selected Areas in Communications, vol. 18, no. 6, pp. 880-890, 2000.

[23] K. Ramchandran, A. Ortega, and M. Vetterli, "Bit allocation for dependent quantization with applications to multiresolution and MPEG video coders," IEEE Transactions on Image Processing, vol. 3, no. 5, pp. 533-545, 1994.

Yong Pei is currently a Tenure-Track Assistant Professor in the Computer Science and Engineering Department, Wright State University, Dayton, Ohio. Previously he was a Visiting Assistant Professor in the Electrical and Computer Engineering Department, University of Miami, Coral Gables, Fla. He received his B.S. degree in electrical power engineering from Tsinghua University, Beijing, in 1996, and M.S. and Ph.D.

degrees in electrical engineering from Rensselaer Polytechnic Institute, Troy, NY, in 1999 and 2002, respectively. His research interests include information theory, wireless communication systems and networks, and image/video compression and communications. He is a Member of IEEE and ACM.

James W. Modestino received the B.S. degree from Northeastern University, Boston, Mass, in 1962, and the M.S. degree from the University of Pennsylvania, Philadelphia, $\mathrm{Pa}$, in 1964, both in electrical engineering. He also received the M.A. and Ph.D. degrees from Princeton University, Princeton, NJ, in 1968 and 1969, respectively. From 1970 to 1972 , he was an Assistant Professor in the Department of Electri-

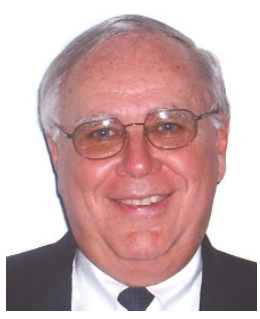
cal Engineering, Northeastern University. In 1972, he joined Rensselaer Polytechnic Institute, Troy, NY, where until leaving in 2001,

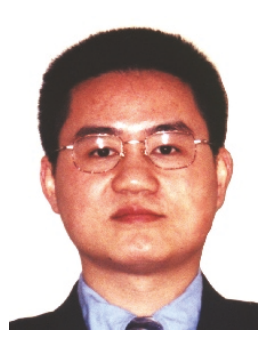

he was an Institute Professor in the Electrical, Computer, and Systems Engineering Department and Director of the Center for Image Processing Research. In 2001, he joined the Department of Electrical and Computer Engineering, University of Miami, Coral Gables, Fla, as the Victor E. Clarke Endowed Scholar, Professor, and Chair. Dr. Modestino is a past Member of the Board of Governors of the IEEE Information Theory Group. He is a past Associate Editor and Book Review Editor for the IEEE Transactions on Information Theory. In 1984, he was corecipient of the Stephen O. Rice Prize Paper Award from the IEEE Communications Society and in 2000 he was corecipient of the Best Paper Award at the International Packet Video Conference. 